\title{
Structured Model Reduction of Interconnected Linear Systems Based on Singular Perturbation
}

\author{
Takayuki Ishizaki $^{\dagger 1}$, Henrik Sandberg ${ }^{2}$, Karl Henrik Johansson ${ }^{2}$, Kenji Kashima ${ }^{3}$, \\ Jun-ichi Imura $^{1}$, Kazuyuki Aihara ${ }^{4}$
}

\begin{abstract}
This paper proposes a singular perturbation approximation that preserves system passivity and an interconnection topology among subsystems. In the first half of this paper, we develop a singular perturbation approximation valid for stable linear systems. Using the relation between the singular perturbation and the reciprocal transformation, we derive a tractable expression of the error system in the Laplace domain, which provides a novel insight to regulate the approximating quality of reduced models. Then in the second half, we develop a structured singular perturbation approximation that focuses on a class of interconnected systems. This structured approximation provides a reduced model that not only possesses fine approximating quality, but also preserves the original interconnection topology and system passivity.
\end{abstract}

\section{INTRODUCTION}

Many of dynamical systems that interest the control community are inherently composed of the interconnection of subsystems. The examples include power networks and transportation networks, as well as control systems in which some controllers are distributed over a plant; see [1], [2] for an overview. Along with the dramatic technical development, the architecture of these interconnected systems has tended to become more complex and larger in scale. In view of this, it is crucial to develop an approximate modeling method to relax the complexity of systems.

Against such a background, this paper develops a model reduction method based on a notion of the singular perturbation approximation, which is one of well-known frameworks to reduce the dynamical complexity of systems. In fact, many of good properties of the singular perturbation approximation, such as the preservation of steady-state distribution and stability preservation under appropriate conditions, have been widely investigated in literature, e.g., [3], [4]. However, the classical singular perturbation theory holds some drawbacks including that:

- the applicability is limited due to the assumption that

\footnotetext{
${ }^{\dagger}$ Research Fellow of the Japan Society for the Promotion of Science

${ }^{1}$ Department of Mechanical and Environmental Informatics, Graduate School of Information Science and Engineering, Tokyo Institute of Technology; 2-12-1, Meguro, Tokyo, Japan:

\{ishizaki@cyb., imura@\}mei.titech.ac.jp

${ }^{2}$ School of Electrical Engineering, Automatic Control, Royal Institute of Technology (KTH), SE-100 44 Stockholm, Sweden:

\{hsan, kallej\}@ee.kth.se

${ }^{3}$ Department of Systems Innovation, Graduate School of Engineering Science, Osaka University; 1-3, Machikaneyama, Toyonaka, Osaka, Japan: kashima@sys.es.osaka-u.ac.jp

${ }^{4}$ Institute of Industrial Science, University of Tokyo; 4-6-1 Komaba, Meguro ward, Tokyo, Japan: aiharaesat.t.u-tokyo.ac.jp
}

systems of interest are intrinsically decoupled into subsystems having different time scales

- the interconnection topology among subsystems is lost through the approximation due to static states to appear in approximants (see Section III-B for details).

As overcoming these drawbacks, we attempt to establish a structured singular perturbation approximation. To this end, we take the following two steps: In the first step, we develop a singular perturbation approximation for general stable linear systems. This is not based on the aforementioned assumption, but by introducing a pre-conditioning coordinate transformation, we decouple a given system into two subsystems having different time scales. The major development here includes the stability analysis of approximants as well as the derivation of a novel error expression in the Laplace domain. Then in the second step, based on the first result, we develop a structured singular perturbation approximation that focuses on a class of interconnected systems. The development includes not only the analysis of passivity preservation but also the preservation of an interconnection structure. In addition, we derive a rigorous $\mathcal{H}_{2}$-error bound of the input-to-output mapping approximation that provides a clear insight to regulate the resultant approximation error. It should be finally remarked that the error analysis in this paper is based on the analysis in the line of our work [5], [6], [7] as well as a relation between the balanced truncation and the reciprocal transformation investigated in [8], [9].

This paper is organized as follows. In Section II, we first develop a singular perturbation approximation for stable linear systems. The major development in the section includes the stability analysis of approximants as well as the derivation of a tractable error expression in the Laplace domain. In Section III, using the result in Section II, we develop a singular perturbation approximation with the preservation of passivity and an interconnection structure among subsystems. In Section IV, we show the efficiency of the proposed approximation through a numerical example, where the reduction of a passive decentralized controller is considered. Finally, concluding remarks are provided in Section V.

NOTATION The following notation is to be used. $\mathbb{R}$ : set of real numbers; $\operatorname{tr}(M)$ : trace of a matrix $M ; \operatorname{im}(M)$ : image of a matrix $M ; \operatorname{diag}\left(M_{1}, \ldots, M_{n}\right)$ : block diagonal matrix having matrices $M_{1}, \ldots, M_{n}$ on its block diagonal. As necessary, $\operatorname{diag}\left(M_{1}, \ldots, M_{n}\right)$ is denoted by $\operatorname{diag}\left(M_{i}\right)_{i \in\{1, \ldots, n\}}$. A matrix $A \in \mathbb{R}^{n \times n}$, not necessarily symmetric, is said to be negative definite (resp. positive definite) if $x^{\top} A x<0$ 
$\left(x^{\top} M x>0\right)$ holds for all $x \neq 0 \in \mathbb{R}^{n}$. The $\mathcal{H}_{\infty}$ and $\mathcal{H}_{2^{-}}$ norm of a stable transfer matrix $G$ are, respectively, denoted by

$$
\begin{aligned}
\|G(s)\|_{\mathcal{H}_{\infty}} & :=\sup _{\omega \in \mathbb{R}}\|G(j \omega)\| \\
\|G(s)\|_{\mathcal{H}_{2}} & :=\left(\frac{1}{2 \pi} \int_{-\infty}^{\infty} \operatorname{tr}\left(G(j \omega) G^{\top}(-j \omega)\right) d \omega\right)^{\frac{1}{2}},
\end{aligned}
$$

where $\|\cdot\|$ denotes the induced 2-norm.

\section{General THEORY}

\section{A. Singular Perturbation Approximation of Linear Systems}

In this section, we first develop a singular perturbation approximation for general stable linear systems. Let us consider a stable linear system

$$
\Sigma:\left\{\begin{array}{l}
\dot{x}=A x+B u \\
y=C x+D u
\end{array}\right.
$$

with $A \in \mathbb{R}^{n \times n}, B \in \mathbb{R}^{n \times m_{u}}, C \in \mathbb{R}^{m_{y} \times n}$ and $D \in$ $\mathbb{R}^{m_{y} \times m_{u}}$, and denote the transfer matrix of $\Sigma$ by $G(s)=$ $C\left(s I_{n}-A\right)^{-1} B+D$, for which we use the notation of

$$
G(s)=\left[\begin{array}{l|l}
A & B \\
\hline C & D
\end{array}\right] .
$$

In much literature on the singular perturbation theory, it is assumed that system (1) is intrinsically decoupled into several subsystems having different time scales; see [3], [4]. Contrastingly, such an assumption is not made in this paper. Instead, by finding an appropriate coordinate transformation, we decouple system (1) into two subsystems in a general manner. More specifically, considering the coordinate transformation of $\Sigma$ by unitary $\left[P^{\top}, Q^{\top}\right]^{\top}$ with $P \in \mathbb{R}^{\hat{n} \times n}$ and $Q \in \mathbb{R}^{(n-\hat{n}) \times n}$, we obtain

$$
\tilde{\Sigma}:\left\{\begin{aligned}
{\left[\begin{array}{c}
\dot{\xi}^{p} \\
\dot{\xi}^{q}
\end{array}\right] } & =\left[\begin{array}{ll}
P A P^{\top} & P A Q^{\top} \\
Q A P^{\top} & Q A Q^{\top}
\end{array}\right]\left[\begin{array}{c}
\xi^{p} \\
\xi^{q}
\end{array}\right]+\left[\begin{array}{c}
P B \\
Q B
\end{array}\right] u \\
y & =\left[\begin{array}{ll}
C P^{\top} & C Q^{\top}
\end{array}\right]\left[\begin{array}{c}
\xi^{p} \\
\xi^{q}
\end{array}\right]+D u
\end{aligned}\right.
$$

To reduce the dimension of $\tilde{\Sigma}$, we impose $\dot{\xi}^{q} \equiv 0$, which means that the behavior of $\xi^{q}$ is to be algebraically determined by $\xi^{p}$ and $u$. Namely, the static state $\hat{\xi}^{q}$, which denotes the approximant of $\xi^{q}$, is constrained by the algebraic equation

$$
\hat{\xi}^{q}=-\left(Q A Q^{\top}\right)^{-1} Q A P^{\top} \hat{\xi}^{p}-\left(Q A Q^{\top}\right)^{-1} Q B u
$$

where the dynamical state $\hat{\xi}^{p}$ is the approximant of $\xi^{p}$ and $Q A Q^{\top}$ is assumed to be non-singular (this assumption is valid if $A$ is negative definite; see Section II-B below for details). This approximation is intuitively reasonable when the convergence rate of $\xi^{q}$ is sufficiently grater than that of $\xi^{p}$. However, it is non-trivial to find such a desirable coordinate transformation.

Substituting (4) into the equation with respect to $\dot{\xi}^{p}$, we have the singular perturbation model

$$
\hat{\Sigma}_{\mathrm{sp}}:\left\{\begin{array}{r}
\dot{\hat{\xi}} p=\hat{A} \hat{\xi}^{p}+\hat{B} u \\
\hat{y}=\hat{C} \hat{\xi}^{p}+\hat{D} u
\end{array}\right.
$$

where

$$
\begin{aligned}
& \hat{A}:=P A P^{\top}-P A \Pi A P^{\top} \in \mathbb{R}^{\hat{n} \times \hat{n}} \\
& \hat{B}:=(P-P A \Pi) B \in \mathbb{R}^{\hat{n} \times m_{u}} \\
& \hat{C}:=C\left(P^{\top}-\Pi A P^{\top}\right) \in \mathbb{R}^{m_{y} \times \hat{n}} \\
& \hat{D}:=D-C \Pi B \in \mathbb{R}^{m_{y} \times m_{u}}
\end{aligned}
$$

and

$$
\Pi:=Q^{\top}\left(Q A Q^{\top}\right)^{-1} Q \in \mathbb{R}^{n \times n} .
$$

Note that this $\Pi$ does not depend on the basis selection of $Q$ because $\Pi=Q^{\top} H^{\top}\left(H Q A Q^{\top} H^{\top}\right)^{-1} H Q$ holds for any unitary matrix $H \in \mathbb{R}^{(n-\hat{n}) \times(n-\hat{n})}$. This fact implies that the singular perturbation model $\hat{\Sigma}_{\mathrm{sp}}$ in (5) depends only on the choice of $P$. Based on the observation above, we define the following terminology:

Definition 1: Consider a transfer matrix $G$ in (2) and let $P \in \mathbb{R}^{\hat{n} \times n}$ such that $P P^{\top}=I_{\hat{n}}$ and $\hat{n} \leq n$. The singular perturbation approximant of $G$ associated with $P$ is defined by

$$
\hat{G}(s ; P):=\left[\begin{array}{l|l}
\hat{A} & \hat{B} \\
\hline \hat{C} & \hat{D}
\end{array}\right]
$$

where $\hat{A}, \hat{B}, \hat{C}$ and $\hat{D}$ are given by (6).

Obviously, the quality of the approximant $\hat{G}$ is dependent on the determination of $P$. In the next subsection, we analyze the property of $\hat{G}$ to construct a reasonable strategy for the determination of $P$.

\section{B. Analysis of Singular Perturbation Approximant}

To analyze the singular perturbation approximant, we introduce a transformation, called the reciprocal transformation [8], [9], as follows:

Definition 2: Consider a transfer matrix $G$ in (2). The reciprocal of $G$ is defined by

$$
G_{-}(s):=\left[\begin{array}{c|c}
A^{-1} & A^{-1} B \\
\hline-C A^{-1} & D-C A^{-1} B
\end{array}\right] .
$$

This reciprocal system satisfies $G\left(s^{-1}\right)=G_{-}(s)$, and some properties of the reciprocal transformation have been investigated in literature; see, e.g., [8], [9]. The following lemma provides a useful relation between the singular perturbation approximation and this reciprocal transformation:

Lemma 1: Given a transfer matrix $G$ in (2) and a matrix $P \in \mathbb{R}^{\hat{n} \times n}$ such that $P P^{\top}=I_{\hat{n}}$ and $\hat{n} \leq n$, let $\hat{G}$ be its singular perturbation approximant associated with $P$ in Definition 1. Then

$$
\hat{G}_{-}(s ; P):=\left[\begin{array}{c|c}
P A^{-1} P^{\top} & P A^{-1} B \\
\hline-C A^{-1} P^{\top} & D-C A^{-1} B
\end{array}\right]
$$

is the reciprocal of $\hat{G}$.

Lemma 1 shows that the reciprocal of the singular perturbation approximant $\hat{G}$ is given by the projection of the reciprocal of $G$ associated with $P$. Note that this lemma can be regarded as a generalization of the results shown in [8], [9], where a relation between the truncation of balanced systems and that of their reciprocal has been investigated. 
From Lemma 1, we obtain the following insight on stability preservation:

Lemma 2: Consider a matrix $P \in \mathbb{R}^{\hat{n} \times n}$ such that $P P^{\top}=$ $I_{\hat{n}}$ and $\hat{n} \leq n$. If $A \in \mathbb{R}^{n \times n}$ is negative definite, then $\hat{A} \in$ $\mathbb{R}^{\hat{n} \times \hat{n}}$ in (6) is negative definite.

Proof: From Lemma 1, we notice that the negative definiteness of $\hat{A}$ is equivalent to that of $P A^{-1} P^{\top}$. Furthermore, the negative definiteness of $P A^{-1} P^{\top}$ is equivalent to that of

$$
P A^{-1} P^{\top}+\left(P A^{-1} P^{\top}\right)^{\top}=P\left(A^{-1}+A^{-\top}\right) P^{\top},
$$

which proves the claim.

Lemma 2 shows that the negative definiteness of $A$, which is a stronger stability condition, is preserved through the singular perturbation approximation. In addition to this, the following lemma ensures the existence of a similarity transformation to make a stable matrix negative definite:

Lemma 3: Given a stable matrix $A \in \mathbb{R}^{n \times n}$, let $V \in$ $\mathbb{R}^{n \times n}$ be a symmetric positive definite matrix such that $A V+$ $V A^{\top}$ is negative definite. Then, $V_{\frac{1}{2}}^{-1} A V_{\frac{1}{2}}$ is negative definite where $V_{\frac{1}{2}}$ is a Cholesky factor of $V$ such that $V=V_{\frac{1}{2}} V_{\frac{1}{2}}^{\top}$.

Lemma 3 shows the existence of a pre-conditioning coordinate transformation to make a stable matrix negative definite. The symmetric positive definite matrix $V$ could be used as a Lyapunov function to prove the stability of $A$. Combining Lemmas 2 and 3, we ensure the stability preservation of the singular perturbation approximation.

Next, we analyze the resultant error of the singular perturbation approximation. In general, the error analysis of the singular perturbation approximation is not necessarily easy due to the complicated form as in (5). To systematically analyze the approximation error, we are required to derive a tractable representation of the error system. In view of this, we derive novel factorization of the error system as follows:

Theorem 1: Given a transfer matrix $G$ in (2) and a matrix $P \in \mathbb{R}^{\hat{n} \times n}$ such that $P P^{\top}=I_{\hat{n}}$ and $\hat{n} \leq n$, let $\hat{G}$ be its singular perturbation approximant in (8). Then, $\hat{G}$ satisfies $\hat{G}(0 ; P)=G(0)$ and

$$
\hat{G}(s ; P)-G(s)=\hat{\Xi}(s ; P) Q^{\top} Q X(s)
$$

where $Q^{\top} Q=I_{n}-P^{\top} P$ and

$$
\begin{aligned}
\hat{\Xi}(s ; P) & :=\left[\begin{array}{c|c}
P A P^{\top}-P A \Pi A P^{\top} & (P-P A \Pi) \\
\hline C\left(P^{\top}-\Pi A P^{\top}\right) & -C \Pi
\end{array}\right] \\
X(s) & :=\left[\begin{array}{c|c}
A & B \\
\hline A & B
\end{array}\right] .
\end{aligned}
$$

In addition, if $A$ is negative definite, then $\hat{G}$ is stable.

Proof: Consider a similarity transformation for the reciprocal $\hat{G}_{-}-G_{-}$of the error system, which is similar to one used in [5], [6], [7]. Then, we have

$$
\hat{G}_{-}(s ; P)-G_{-}(s)=\hat{\Xi}_{-}(s ; P) Q^{\top} Q X_{-}(s)
$$

where

$$
\begin{aligned}
\hat{\Xi}_{-}(s ; P) & :=\left[\begin{array}{c|c}
P A^{-1} P^{\top} & P A^{-1} \\
\hline-C A^{-1} P^{\top} & -C A^{-1}
\end{array}\right] \\
X_{-}(s) & :=\left[\begin{array}{c|c}
A^{-1} & A^{-1} B \\
\hline-I_{n} & 0
\end{array}\right] .
\end{aligned}
$$

Replacing $B$ and $D$ with $I_{n}$ and 0 , respectively, in (10), we notice that $\hat{\Xi}_{-}$is the reciprocal of $\hat{\Xi}$ in (12). Hence, from Lemma 1 and

$$
\begin{aligned}
\hat{G}(s ; P)-G(s) & =\hat{G}_{-}\left(s^{-1} ; P\right)-G_{-}\left(s^{-1}\right) \\
& =\hat{\Xi}_{-}\left(s^{-1} ; P\right) Q^{\top} Q X_{-}\left(s^{-1}\right) \\
& =\hat{\Xi}(s ; P) Q^{\top} Q X(s),
\end{aligned}
$$

the factorization (11) follows. In addition, substituting $s=0$ into (11), we notice that $\hat{G}(0 ; P)=G(0)$ because $X(0)=0$. Finally, if $A$ is negative definite, $\hat{A}$ is also negative definite as shown in Lemma 2. Hence, the stability of $\hat{G}$ follows.

This factorization of the error system provides an insight that the singular perturbation approximation works well if the norm of $Q X$ is sufficiently small, where $Q$ denotes an orthogonal complement of $P$. It should be noted that $X$ in (12) coincides with the transfer matrix from $u$ to $\dot{x}$ of the original system $\Sigma$, and $\hat{\Xi}$ coincides with the singular perturbation approximant of

$$
\Xi(s):=\left[\begin{array}{c|c}
A & I_{n} \\
\hline C & 0
\end{array}\right]
$$

associated with $P$.

\section{Structured Singular Perturbation}

\section{A. Passivity Preservation}

In this section, based on the result in Section II, we develop a structured singular perturbation approximation that is specialized for a class of interconnected systems. Generally speaking, properties of interconnected systems, such as stability, are not straightforwardly characterized by those of local subsystems. This fact often inhibits to analyze interconnected systems locally. On the other hand, it is well known that interconnected systems composed of the negative feedback interconnection of passive subsystems are passive [10], [11]. This implies that the local analysis of subsystems is also valid for interconnected ones. In this sense, the system passivity is one of key properties to analyze and synthesize interconnected systems.

In view of this, we develop a singular perturbation approximation that preserves system passivity. To this end, we introduce the standard passivity of systems as follows [12], [13]:

Definition 3: A linear system $\Sigma$ in (1) is said to be $V$ passive if there exists a symmetric positive definite matrix $V$ such that

$$
\mathcal{S}(A, B, C, D ; V):=\left[\begin{array}{cc}
A V+V A^{\top} & V C^{\top}-B \\
C V-B^{\top} & -D-D^{\top}
\end{array}\right]
$$

is negative definite. 
Similarly to Lemma 3, we consider the following coordinate transformation of passive systems:

Lemma 4: For any $V$-passive system $\Sigma$ in (1)

$$
\mathcal{S}\left(V_{\frac{1}{2}}^{-1} A V_{\frac{1}{2}}, V_{\frac{1}{2}}^{-1} B, C V_{\frac{1}{2}}, D ; I_{n}\right)
$$

is negative definite, where $V_{\frac{1}{2}}$ is a Cholesky factor of $V$ such that $V=V_{\frac{1}{2}} V_{\frac{1}{2}}^{\top}$.

Proof: The claim follows from the fact that the matrix in (15) is rewritten as

$$
\tilde{V} \mathcal{S}\left(V_{\frac{1}{2}}^{-1} A V_{\frac{1}{2}}, V_{\frac{1}{2}}^{-1} B, C V_{\frac{1}{2}}, D ; I_{n}\right) \tilde{V}^{\top}
$$

where $\tilde{V}=\operatorname{diag}\left(V_{\frac{1}{2}}, I_{m_{u}}\right)$.

This coordinate transformation is useful because the unit matrix $I_{n}$ is the solution of the matrix inequality of (15). A similar realization has appeared in [10], and is called a selfdual realization. From this lemma, we can assume, without loss of generality, that any $V$-passive system is $I_{n}$-passive. Based on this, we obtain the following theorem:

Theorem 2: Let an $I_{n}$-passive system $\Sigma$ in (1) and let $P \in \mathbb{R}^{\hat{n} \times n}$ such that $P P^{\top}=I_{\hat{n}}$ and $\hat{n} \leq n$. If

$$
\operatorname{im}\left(\left[B, C^{\top}\right]\right) \subseteq \operatorname{im}\left(P^{\top}\right)
$$

holds, then the singular perturbation model $\hat{\Sigma}_{\mathrm{sp}}$ in (5) is $I_{\hat{n}^{-}}$ passive.

Proof: We use the fact that $\hat{A}$ can be factorized as

$$
\hat{A}=(P-P A \Pi) A(P-P A \Pi)^{\top} \text {; }
$$

see [14] for a proof. Noting that $\hat{B}=P B$ and $\hat{C}=C P^{\top}$ hold by the assumption of $Q B=0$ and $C Q^{\top}=0$, we verify that $\mathcal{S}\left(\hat{A}, \hat{B}, \hat{C}, \hat{D} ; I_{\hat{n}}\right)=\tilde{P} \mathcal{S}\left(A, B, C, D ; I_{n}\right) \tilde{P}^{\top}$ holds where $\tilde{P}=\operatorname{diag}\left(P-P A \Pi, I_{m_{u}}\right)$. This proves the claim.

This theorem shows that the singular perturbation approximation of $I_{n}$-passive systems appropriately preserves the passivity as long as (16) holds.

\section{B. Preservation of Decentralized Feedback Interconnection}

In this subsection, we focus on a class of interconnected systems and investigate a condition to preserve the interconnection topology. The singular perturbation approximation, in general, yields dense system matrices in (6) even if the original system matrices have some sparsity representing an interconnection topology of subsystems. This means that the interconnection topology of the original system is extinguished through the approximation. To preserve this, it is essential that we introduce suitable sparsity of $P$ compatible with sparsity of the system matrices.

In the rest of this paper, we focus on the following class of interconnected systems:

$$
\begin{aligned}
& \Sigma_{0}:\left\{\begin{aligned}
\dot{x}_{0} & =A_{0} x_{0}+B_{0} u+\sum_{l=1}^{L} b_{0, l} w_{l} \\
y & =C_{0} x_{0}+D_{0} u \\
z_{l} & =c_{0, l} x_{0}
\end{aligned}\right. \\
& \Sigma_{l}:\left\{\begin{array}{l}
\dot{x}_{l}=A_{l} x_{l}+b_{l} z_{l} \\
w_{l}=-\left(c_{l} x_{l}+d_{l} z_{l}\right), \quad l \in \mathbb{L}
\end{array}\right.
\end{aligned}
$$

where $\mathbb{L}:=\{1, \ldots, L\}$. For simplicity of notation, we omit each matrix dimension and assume that all quantities have compatible dimension. The structure of (17) represents a decentralized negative feedback interconnection of subsystems $\Sigma_{l}$ to the hub subsystem $\Sigma_{0}$. Such a structure appears in decentrally controlled systems, where $\Sigma_{0}$ and $\Sigma_{l}$ can be regarded as a plant and decentralized controllers, respectively.

The interconnected system can be rewritten by the structured system matrices of

$$
\begin{aligned}
& A=\left[\begin{array}{cc}
\operatorname{diag}\left(A_{l}\right)_{l \in \mathbb{L}} & \operatorname{diag}\left(b_{l}\right)_{l \in \mathbb{L}} c_{0, \mathbb{L}} \\
-b_{0, \mathbb{L}} \operatorname{diag}\left(c_{l}\right)_{l \in \mathbb{L}} & A_{0}-b_{0, \mathbb{L}} \operatorname{diag}\left(d_{l}\right)_{l \in \mathbb{L} c_{0, \mathbb{L}}}
\end{array}\right] \\
& B=\left[\begin{array}{c}
0 \\
B_{0}
\end{array}\right], C=\left[\begin{array}{ll}
0 & C_{0}
\end{array}\right], D=D_{0}
\end{aligned}
$$

where $b_{0, \mathbb{L}}:=\left[b_{0,1}, \ldots, b_{0, L}\right]$ and $c_{0, \mathbb{L}}:=\left[c_{0,1}^{\top}, \ldots, c_{0, L}^{\top}\right]^{\top}$. For convenience, we define the following terminology:

Definition 4: A linear system $\Sigma$ in (1) is said to be a decentrally interconnected network if $A, B, C$ and $D$ are in the form of (18).

It should be emphasized that in order to preserve the structure in Definition 4, we are required to take into account sparsity of $P$ as well as that of $Q$ because $\Pi$ in (7) possibly becomes a dense matrix due to the inversion $\left(Q A Q^{\top}\right)^{-1}$. To realize suitable sparsity of the inversion, compatible with the structure in (18), we impose the following specific structure on $P$ :

Definition 5: Let be given a decentrally interconnected network $\Sigma$ in Definition 4. An aggregation matrix compatible with (18) is defined by

$$
P:=\operatorname{diag}\left(p_{1}, \ldots, p_{L}, I_{n_{0}}\right) \in \mathbb{R}^{\hat{n} \times n}
$$

where each $p_{l} \in \mathbb{R}^{\hat{n}_{l} \times n_{l}}$ satisfies $p_{l} p_{l}^{\top}=I_{\hat{n}_{l}}$ and $\sum_{l=1}^{L} \hat{n}_{l}+$ $n_{0}=\hat{n}$.

This structure of $P$ implies that the singular perturbation approximation is applied with respect to each subsystem. Hereafter, we denote an orthogonal complement of $p_{l} \in$ $\mathbb{R}^{\hat{n}_{l} \times n_{l}}$ by $q_{l} \in \mathbb{R}^{\left(n_{l}-\hat{n}_{l}\right) \times n_{l}}$, i.e., $p_{l}^{\top} p_{l}+q_{l}^{\top} q_{l}=I_{n_{l}}$ holds for each $l \in \mathbb{L}$. Based on the formulation above, we obtain the following theorem:

Theorem 3: Let be given a decentrally interconnected network $\Sigma$ in Definition 4. For any aggregation matrix $P$ in Definition 5 , the singular perturbation model $\hat{\Sigma}_{\mathrm{sp}}$ is again a decentrally interconnected network and is given by the system matrices of

$$
\begin{aligned}
& \hat{A}=\left[\begin{array}{cc}
\operatorname{diag}\left(\hat{A}_{l}\right)_{l \in \mathbb{L}} & \operatorname{diag}\left(\hat{b}_{l}\right)_{l \in \mathbb{L}} c_{0, \mathbb{L}} \\
-b_{0, \mathbb{L}} \operatorname{diag}\left(\hat{c}_{l}\right)_{l \in \mathbb{L}} & A_{0}-b_{0, \mathbb{L}} \operatorname{diag}\left(\hat{d}_{l}\right)_{l \in \mathbb{L}} c_{0, \mathbb{L}}
\end{array}\right] \\
& \hat{B}=\left[\begin{array}{c}
0 \\
B_{0}
\end{array}\right], \quad \hat{C}=\left[\begin{array}{ll}
0 & C_{0}
\end{array}\right], \quad \hat{D}=D_{0}
\end{aligned}
$$

where

$$
\begin{array}{ll}
\hat{A}_{l}:=p_{l} A_{l} p_{l}^{\top}-p_{l} A_{l} \pi_{l} A_{l} p_{l}^{\top}, & \hat{b}_{l}:=\left(p_{l}-p_{l} A_{l} \pi_{l}\right) b_{l} \\
\hat{c}_{l}:=c_{l}\left(p_{l}^{\top}-\pi_{l} A_{l} p_{l}^{\top}\right), & \hat{d}_{l}:=d_{l}-c_{l} \pi_{l} b_{l}
\end{array}
$$


and $\pi_{l}:=q_{l}^{\top}\left(q_{l} A_{l} q_{l}^{\top}\right)^{-1} q_{l}$.

Proof: Note that the structure of the aggregation matrix $P$ allows that its orthogonal complement is in the form of

$$
Q=\left[\operatorname{diag}\left(q_{1}, \ldots, q_{L}\right), 0\right] \in \mathbb{R}^{(n-\hat{n}) \times n} .
$$

Thus, it is readily verified that $\hat{B}=P B, \hat{C}=C P^{\top}$ and $\hat{D}=D$ hold due to $Q B=0$ and $C Q^{\top}=0$. This proves the claim for $\hat{B}, \hat{C}$ and $\hat{D}$ in (20). In addition, the specific structure of $P$ and $Q$ yields

$$
\begin{aligned}
P A P^{\top} & =\left[\begin{array}{cc}
\operatorname{diag}\left(p_{l} A_{l} p_{l}^{\top}\right)_{l \in \mathbb{L}} & \operatorname{diag}\left(p_{l} b_{l}\right)_{l \in \mathbb{L}} c_{0, \mathbb{L}} \\
-b_{0, \mathbb{L}} \operatorname{diag}\left(c_{l} p_{l}^{\top}\right)_{l \in \mathbb{L}} & A_{0}-b_{0, \mathbb{L}} \operatorname{diag}\left(d_{l}\right)_{l \in \mathbb{L}} c_{0, \mathbb{L}}
\end{array}\right] \\
P A Q^{\top} & =\left[\begin{array}{c}
\operatorname{diag}\left(p_{l} A_{l} q_{l}^{\top}\right)_{l \in \mathbb{L}} \\
-b_{0, \mathbb{L}} \operatorname{diag}\left(c_{l} q_{l}^{\top}\right)_{l \in \mathbb{L}}
\end{array}\right] \\
Q A P^{\top} & =\left[\begin{array}{ll}
\operatorname{diag}\left(q_{l} A_{l} p_{l}^{\top}\right)_{l \in \mathbb{L}} & \operatorname{diag}\left(q_{l} b_{l}\right)_{l \in \mathbb{L} c_{0, \mathbb{L}}}
\end{array}\right] \\
Q A Q^{\top} & =\operatorname{diag}\left(q_{l} A_{l} q_{l}^{\top}\right)_{l \in \mathbb{L} .}
\end{aligned}
$$

Note that $\left(Q A Q^{\top}\right)^{-1}=\operatorname{diag}\left(\left(q_{l} A_{l} q_{l}^{\top}\right)^{-1}\right)_{l \in \mathbb{L}}$ holds. Thus, $\hat{A}$ in (6) is given by (20).

Theorem 3 shows that the singular perturbation approximation associated with the aggregation matrix $P$ appropriately preserves the decentralized interconnection of the original system. In addition, comparing (20) with (18), we notice that the singular perturbation approximation associated with $P$ in (19) just coincides with that of each subsystem associated with $p_{l}$, and also that the dynamics of the hub subsystem is exactly left through the approximation.

\section{Approximation of Decentrally Interconnected Networks composed of Passive Subsystems}

In this subsection, combining all results above, we propose a structured singular perturbation approximation of decentrally interconnected networks. For convenience, we define $\hat{\Sigma}_{l}$ by replacing $A_{l}, b_{l}, c_{l}$ and $d_{l}$ in (17) with $\hat{A}_{l}, \hat{b}_{l}, \hat{c}_{l}$ and $\hat{d}_{l}$ in (21). The following theorem provides an $\mathcal{H}_{2}$-error bound of the structured approximation, where the principal submatrix of $M$ corresponding to the $l$-th subsystem is denoted by $[M]_{l}$, e.g., $[A]_{l}=A_{l}$ holds for $A$ in (18):

Theorem 4: Let be given a decentrally interconnected network $\Sigma$ in Definition 4 and assume that

$$
\Sigma_{0-z, w}:\left\{\begin{aligned}
\dot{x}_{0} & =A_{0} x_{0}+b_{0, \mathbb{L}} w \\
z & =c_{0, \mathbb{L}} x_{0}
\end{aligned}\right.
$$

is $V_{0}$-passive and all subsystems $\Sigma_{l}$ in (17) are $I_{n_{l}}$-passive. Let $G$ in (2) be the transfer matrix of $\Sigma$ and $\Phi \in \mathbb{R}^{n \times n}$ such that $\Phi A+A \Phi^{\top}+B B^{\top}=0$. If the aggregation matrix $P \in$ $\mathbb{R}^{\hat{n} \times n}$ in Definition 5 satisfies that $\operatorname{im}\left(\left[b_{l}, c_{l}^{\top}\right]\right) \subseteq \operatorname{im}\left(p_{l}^{\top}\right)$ and

$$
q_{l}\left[A \Phi A^{\top}\right]_{l} q_{l}^{\top}-\operatorname{diag}\left(\lambda_{l}^{1}, \ldots, \lambda_{l}^{n_{l}-\hat{n}_{l}}\right)
$$

is negative semidefinite for each $l \in \mathbb{L}$, then the singular perturbation model $\hat{\Sigma}_{\text {sp }}$ in (5) is a stable decentrally interconnected network, which is composed of $V_{0}$-passive $\Sigma_{0-z, w}$ and $I_{\hat{n}_{l}}$-passive $\hat{\Sigma}_{l}$ for $l \in \mathbb{L}$, and satisfies $\hat{G}(0 ; P)=G(0)$ and

$$
\|\hat{G}(s ; P)-G(s)\|_{\mathcal{H}_{2}} \leq\|\hat{\Xi}(s ; P)\|_{\mathcal{H}_{\infty}} \sqrt{\sum_{l=1}^{L} \sum_{i=1}^{n_{l}-\hat{n}_{l}} \lambda_{l}^{i}}
$$

where $\hat{\Xi}$ is defined in (12).

Proof: Theorem 3 shows that the singular perturbation approximation associated with $P$ in (19) just coincides with that of each subsystem associated with $p_{l}$. In addition, from Theorem 2 with $\operatorname{im}\left(\left[b_{l}, c_{l}^{\top}\right]\right) \subseteq \operatorname{im}\left(p_{l}^{\top}\right)$, the singular perturbation approximation of each $I_{n_{l}}$-passive $\Sigma_{l}$ yields $I_{\hat{n}_{l}}$-passive approximants $\hat{\Sigma}_{l}$. Thus, $\hat{\Sigma}_{\mathrm{sp}}$, which consists of the negative feedback interconnection of $V_{0}$ - and $I_{\hat{n}_{l}}$-passive subsystems, is stable. Furthermore, using (11), we have

$$
\|\hat{G}(s ; P)-G(s)\|_{\mathcal{H}_{2}} \leq\|\hat{\Xi}(s ; P)\|_{\mathcal{H}_{\infty}}\|Q X(s)\|_{\mathcal{H}_{2}}
$$

where $Q$ is given by (22). Note that $Q B=0$ holds, which implies that the feedthrough term of $Q X$ is null. Thus, $\|Q X\|_{\mathcal{H}_{2}}$ is bounded and is given by

$$
\|Q X(s)\|_{\mathcal{H}_{2}}^{2}=\operatorname{tr}\left(Q A \Phi A^{\top} Q^{\top}\right),
$$

which is upper bounded by $\sum_{l=1}^{L} \sum_{i=1}^{n_{l}-\hat{n}_{l}} \lambda_{l}^{i}$ due to (24). This proves the claim.

This theorem shows that the structured approximation works well if the sum of eigenvalues of $\left[A \Phi A^{\top}\right]_{l}$ that are neglected through the approximation is small enough. Based on the error analysis above, we provide the following algorithm for systematic reduction, where we denote $\mathbb{N}_{l}:=$ $\left\{1, \ldots, n_{l}\right\}$ :

(a) Prescribe a threshold $\epsilon$ and let null matrices $\eta_{l}$ for $l \in \mathbb{L}$.

(b) Calculate the index matrix $A \Phi A^{\top}$ from $(A, B)$.

(c) Find the set $\left\{\left(\lambda_{l}^{i}, v_{l}^{i}\right)\right\}_{i \in \mathbb{N}_{l}}$ of all eigenpairs of the diagonal blocks $\left[A \Phi A^{\top}\right]_{l}$ for each $l \in \mathbb{L}$, where the eigenvectors $v_{l}^{i}$ are normalized as $\left\|v_{l}^{i}\right\|=1$.

(d) For $l \in \mathbb{L}$ and $i \in \mathbb{N}_{l}$, update $\eta_{l} \leftarrow\left[\eta_{l}, v_{l}^{i}\right]$ if $\lambda_{l}^{i} \geq \epsilon$.

(e) Find $p_{l}$ such that $\operatorname{im}\left(p_{l}^{\top}\right)=\operatorname{im}\left(\left[\eta_{l}, b_{l}, c_{l}^{\top}\right]\right)$ and $p_{l} p_{l}^{\top}=$ $I_{\hat{n}_{l}}$ by the Gram-Schmidt process.

(f) Construct the singular perturbation model in (5) associated with the aggregation matrix $P$ in (19).

This algorithm finds $p_{l} \in \mathbb{R}^{\hat{n}_{l} \times n_{l}}$ such that $\lambda_{l}^{i}<\epsilon$ for all $i \in\left\{1, \ldots, n_{l}-\hat{n}_{l}\right\}$ in (24). Note that for the construction of each $p_{l}$, we only need the eigenvalue decomposition and the Gram-Schmidt process for matrices with the dimension $n_{l}$ of each subsystem.

\section{NUMERICAL EXAMPLE}

We show the efficiency of the proposed structured approximation through a numerical example. Let us consider the $2 \nu$-dimensional mass-spring-damper system

$$
M \ddot{q}+L \dot{q}+K q=F w, \quad y=z=F^{\top} \dot{q}
$$

with $\nu=50, M=I_{\nu}, L=(1 / 4) I_{\nu}$ and

$$
K=\left[\begin{array}{cccc}
2 & -1 & & \\
-1 & 2 & \ddots & \\
& \ddots & \ddots & -1 \\
& & -1 & 2
\end{array}\right], \quad F=\left[\begin{array}{cc}
1 & 0 \\
0 & \vdots \\
\vdots & 0 \\
0 & 1
\end{array}\right]
$$

A depiction of this system is given in Fig. 1, where we use the notation $w=\left[w_{1}, w_{2}\right]^{\top}, y=\left[y_{1}, y_{2}\right]^{\top}, z=\left[z_{1}, z_{2}\right]^{\top}$ and $q=\left[q_{1}, \ldots, q_{50}\right]^{\top}$. 


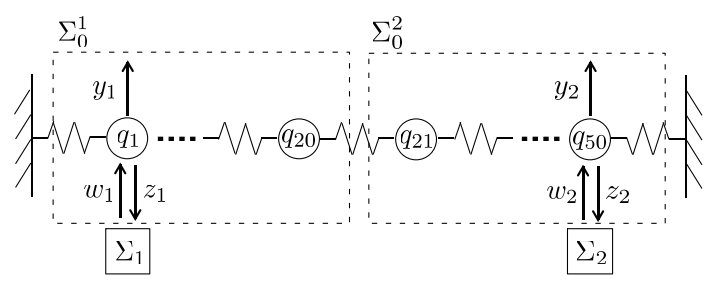

Fig. 1. Depiction of Mass-Spring-Damper System with Decentralized Control.

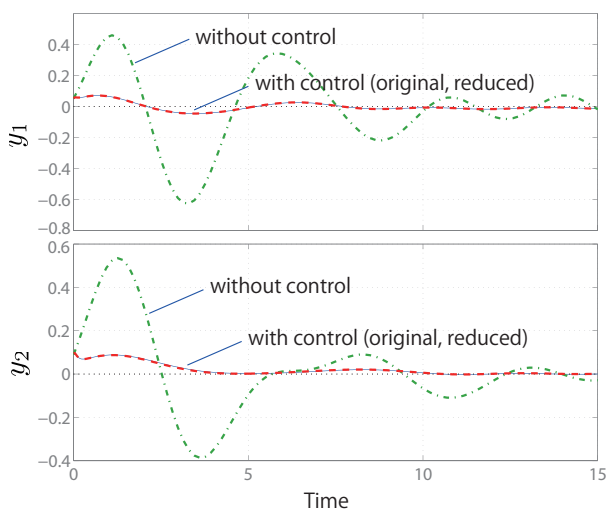

Fig. 2. Trajectory of Output $y$.

By letting $x_{0}:=\left[q^{\top}, \dot{q}^{\top}\right]^{\top} \in \mathbb{R}^{2 \nu}$, we have the 100dimensional plant $\Sigma_{0-z, w}$ in (23) with

$$
A_{0}=\left[\begin{array}{cc}
0 & I_{\nu} \\
-M^{-1} K & -M^{-1} L
\end{array}\right], \quad b_{0, \mathbb{L}}=\left[\begin{array}{c}
0 \\
M^{-1} F
\end{array}\right]
$$

and $c_{0, \mathbb{L}}=\left[0, F^{\top}\right]$. As shown in [10], this $\Sigma_{0-z, w}$ is $V_{0^{-}}$ passive with $V_{0}=\operatorname{diag}\left(K^{-1}, M^{-1}\right)$. For this plant, we construct a decentralized controller composed of passive local controllers $\Sigma_{l}$ in (17) for $l \in\{1,2\}$. Here, we apply the design technique of a centralized passive controller proposed in [11] to each truncated (disconnected) sub-plant denoted by $\Sigma_{0}^{l}$ in Fig. 1. As a result, we obtain a 100dimensional decentralized passive controller. In Fig. 2, the output trajectories with and without control are shown by the solid and chain lines, respectively, where the initial condition $x_{0}(0)$ of the plant is given randomly.

Next, supposing that the performance of the decentralized controller is desirable, we reduce the dimension of each controller by using our structured approximation. To guarantee the performance for any initial condition $x_{0}(0)$, we apply the dual counterpart of Theorem 4, namely we approximate the state-to-output mapping defined by $(A, C)$ in (18) with fixed $C_{0}=\left[0, F^{\top}\right]$. Assigning the threshold in the algorithm in Section III-C as $\epsilon=10^{-3}$, both controllers are reduced to 5-dimensional ones, which preserves the decentralized feedback interconnection as well as passivity. The output trajectory of the controlled system with the reduced order controllers is shown also in Fig. 2 by the dot lines. From this result, we can see that the dimension of the passive controllers is appropriately reduced almost without degrading the performance.

\section{CONCLUSion}

In this paper, we have proposed a singular perturbation approximation with the preservation of passivity and an interconnection topology. First, investigating the relation between the singular perturbation and the reciprocal transformation, we have derived useful factorization of the error system. In the second half of this paper, based on the result in the first half, we have established a network structure preserving approximation for a class of interconnected systems. Our approximation procedure produces a reduced model that not only possesses fine approximating quality but also preserves the original interconnection topology and system passivity.

\section{ACKNOWLEDGMENT}

This research is partially supported by the Aihara Innovative Mathematical Modelling Project, the Japan Society for the Promotion of Science (JSPS) through the "Funding Program for World-Leading Innovative R\&D on Science and Technology (FIRST Program)," initiated by the Council for Science and Technology Policy (CSTP).

\section{REFERENCES}

[1] S. Boccaletti, V. Latora, Y. Moreno, M. Chavez, and D. U. Hwang, "Complex networks: Structure and dynamics," Physics Reports, vol. 424-4-5, pp. 175-308, 2006.

[2] M. Mesbahi and M. Egerstedt, Graph Theoretic Methods in Multiagent Networks. Princeton Univerwity Press, 2010.

[3] P. V. Kokotovic, H. K. Khalil, and J. O'reilly, Singular perturbation methods in control: analysis and design. Society for Industrial Mathematics, 1999.

[4] H. K. Khalil and J. W. Grizzle, Nonlinear systems. Macmillan Publishing Company New York, 1992.

[5] T. Ishizaki, K. Kashima, J. Imura, and K. Aihara, "Reaction-diffusion clustering of single-input dynamical networks," in 50th IEEE Conference on Decision and Control, and European Control Conference, 2011, pp. 7837-7842.

[6] — , "Model reduction of multi-input dynamical networks based on clusterwise controllability," in Proceedings of American Control Conference, 2012, pp. 2301-2306.

[7] T. Ishizaki, K. Kashima, A. Girard, J. Imura, L. Chen, and K. Aihara, "Clustering-based $\mathcal{H}_{2}$-state aggregation of positive networks and its application to reduction of chemical master equation," in 51st IEEE Conference on Decision and Control, 2012 (to appear).

[8] K. V. Fernando and H. Nicholson, "Reciprocal transformations in balanced model-order reduction," Control Theory and Applications, IEE Proceedings D, vol. 130-6, pp. 359-362, 1983.

[9] Y. Liu and B. D. O. Anderson, "Singular perturbation approximation of balanced systems," in 28th IEEE Conference on Decision and Control, 1989, pp. 1355-1360.

[10] W. M. Haddad, S. B. Dennis, and Y. W. Wang, "Dissipative $H_{2} / H_{\infty}$ controller synthesis," IEEE Transactions on Automatic Control, vol. 39-4, pp. 827-831, 1994.

[11] R. Lozano-Leal, "On the design of dissipative LQG-type controller," in Proc. of the 27th IEEE Conference on Decision and Control, 1988, pp. $1645-1646$.

[12] A. C. Antoulas, Approximation of Large-Scale Dynamical Systems. Society for Industrial Mathematics, 2005.

[13] L. Knockaert, "A note on strict passivity," Systems \& Control Letters, vol. 54, pp. 865-869, 2005.

[14] T. Ishizaki, H. Sandberg, K. H. Johansson, K. Kashima, J. Imura, and K. Aihara, "Singular perturbation approximation of semistable linear systems," in Proc. of 2013 European Control Conference, 2013 (to appear). 